\title{
Ileitis Is Not Always Crohn's Disease: Case Report
}

\author{
Abdel Rahman Omer ${ }^{\mathrm{a}}$, Omar Nadhem ${ }^{\mathrm{a}, \mathrm{b}}$, Nibras Talibmamury ${ }^{\mathrm{a}}$, \\ Yasir Al Abboodia , James Lusby ${ }^{\text {a }}$, Steven Urban ${ }^{\mathrm{a}}$
}

\begin{abstract}
Eosinophilic gastroenteritis (EG) is a rare disorder characterized by eosinophilic infiltration of the bowel wall with various gastrointestinal manifestations. Clinical presentation may vary depending on sites and depth of involvement of the gastrointestinal tract. Imaging has very little role in supporting the diagnosis, and therefore a high degree of clinical suspicion is required. In this article, we describe a 51-year-old male who presented with severe abdominal pain, nausea, vomiting and constipation. CT scan of the abdomen showed ileitis and laproscopy revealed ileitis with stricture in the terminal ileum. The patient was treated with laproscopic right hemicolectomy with final diagnosis of EG.
\end{abstract}

Keywords: Eosinophilic gastroenteritis; Muscularispropria; Small bowel obstruction

\section{Introduction}

Eosinophilic gastroenteritis (EG) is a rare disorder characterized by eosinophilic infiltration of the bowel wall with various gastrointestinal manifestations [1]. The involvement of different layers of the intestinal wall usually gives rise to different clinical manifestations. The mucosal form, which is the most common EG subtype, presents with vomiting, abdominal pain, diarrhea, fecal blood loss, anemia and weight loss secondary to malabsorption. The muscularis form is characterized by infiltration of eosinophils predominantly in the muscularis layer, leading to thickening of the bowel wall, which might result in gastrointestinal obstructive symptoms. The subserosal form occurs in a minority of patients with

Manuscript accepted for publication July 29, 2014

${ }^{a}$ Internal Medicine Department, Texas Tech University Health Sciences Center, Amarillo, TX, USA

${ }^{b}$ Corresponding Author: Omar Nadhem, Internal Medicine Department, Texas Tech University Health Sciences Center, 1400 Coulter, Amarillo, TX 79106, USA. Email: omar.nadhem@ttuhsc.edu

doi: http://dx.doi.org/10.14740/jmc1869w
$\mathrm{EG}$, and it is characterized by exudative ascites with higher peripheral eosinophil counts compared with the other forms [2]. The etiology of EG is unknown and its pathogenesis is poorly understood. EG has features of allergy and immune dysregulation but does not clearly fit into the category of allergic or immune disorder [3]. The diagnostic criteria for the EG are well defined. They include gastrointestinal symptoms, eosinophilic infiltration in at least one area of the digestive tract demonstrated by biopsy, absence of eosinophilic infiltration in other organs outside the digestive tract and absence of parasitic infections [4]. Corticosteroids are the mainstay of therapy with a $90 \%$ response rate in some studies. Appropriate duration of steroid treatment is unknown and relapse often necessitates long-term treatment [1].

\section{Case Report}

We present a 51-year old white gentleman who presented with acute onset of severe constant right-sided abdominal pain associated with abdominal distension, nausea and vomiting. He was unable to tolerate solids or liquids. He states that he has not had a bowel movement or passed flatus in the last $48 \mathrm{~h}$. He had a colonoscopy 5 months prior to this presentation which showed moderately severe diverticulosis of the sigmoid colon. Past surgical history consists of inguinal hernia repair and appendectomy.

\section{On examination}

He was in mild distress, and abdomen was soft, distended with tenderness in the right lower quadrant and otherwise normal exam.

\section{Labs}

WBC $11,800 / \mu \mathrm{L}$ with $81 \%$ neutrophils and $0.7 \%$ eosinophils, HGB $14.8 \mathrm{~g} / \mathrm{dL}$, platelets $250,000 / \mu \mathrm{L}$. Sodium 140 $\mathrm{mmol} / \mathrm{L}$, potassium $3.9 \mathrm{mmol} / \mathrm{L}$, chloride $107 \mathrm{mmol} / \mathrm{L}, \mathrm{CO}_{2}$ $26 \mathrm{mmol} / \mathrm{L}$, glucose $137 \mathrm{mg} / \mathrm{dL}$, BUN $10 \mathrm{mg} / \mathrm{dL}$, creatinine $1.0 \mathrm{mg} / \mathrm{dL}$, total bilirubin $0.8 \mathrm{mg} / \mathrm{dL}$, total protein $6.1 \mathrm{~g} / \mathrm{dL}$, albumin $3.6 \mathrm{~g} / \mathrm{dL}$, alkaline phosphatase $52 \mathrm{IU} / \mathrm{L}$, SGOT 20 


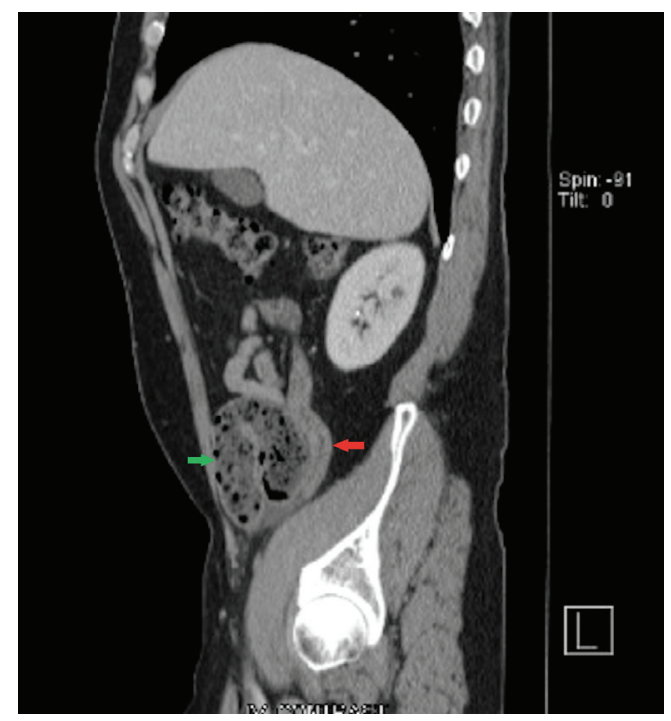

Figure 1. CT of abdomen and pelvis with intravenous contrast showed bowel wall thickening and inflammatory changes around the terminal ileum. Red arrow indicates bowel wall thickening and inflammatory changes around the terminal ileum (sagittal view). Green arrow indicates dilatation of the small bowel just proximal to ileitis area (sagittal view).

\section{IU/L and SGPT $20 \mathrm{IU} / \mathrm{L}$.}

\section{Imaging}

$\mathrm{CT}$ of abdomen and pelvis with intravenous contrast showed bowel wall thickening and inflammatory changes around the terminal ileum (Fig. 1). Findings suggest inflammatory bowel disease or infectious enteritis. Mild dilatation of the small bowel just proximal to this area may be a reactive ileus. Sigmoid and descending colon diverticulosis without features of acute diverticulitis are present.

\section{Hospital course}

The patient was kept nothing per mouth with intravenous fluids and intravenous analgesia. Surgery team was consulted and due to the severity of symptoms they decided to proceed with laproscopic procedure. During the procedure, the ileum was distended with thickened bowel wall. Several centimeters proximal to the ileocecal valve, there was a puckering which appeared to be stricture with some ulceration with no obvious cobble stoning. The colonic mucosa was tan-pink to red, smooth and glistening, and displays prominent irregular folds. Right hemicolectomy was done and the tissue was sent for pathologic evaluation.

\section{Pathology}

Dense infiltrate of eosinophils in the muscularis propria suggests EG (Fig. 2). Small areas of ischemic damage are present. Neither granulomas nor parasitic forms are identified.

\section{Post-procedure course}

The patient had a prolonged postoperative ileus requiring TPN for few days; however, his ileus ultimately did resolve and he was able to tolerate his diet. He was discharged home later on.

\section{Discussion}

EG is a rare, chronic relapsing inflammatory disease of the bowel characterized by the presence of peripheral eosinophilia and eosinophilic infiltration of one or more layers of the intestinal wall [5]. Clinical features depend upon the most prominent layer of visceral wall involvement by eosinophils, i.e. mucosal, muscular or serosal [6]. Approxi-
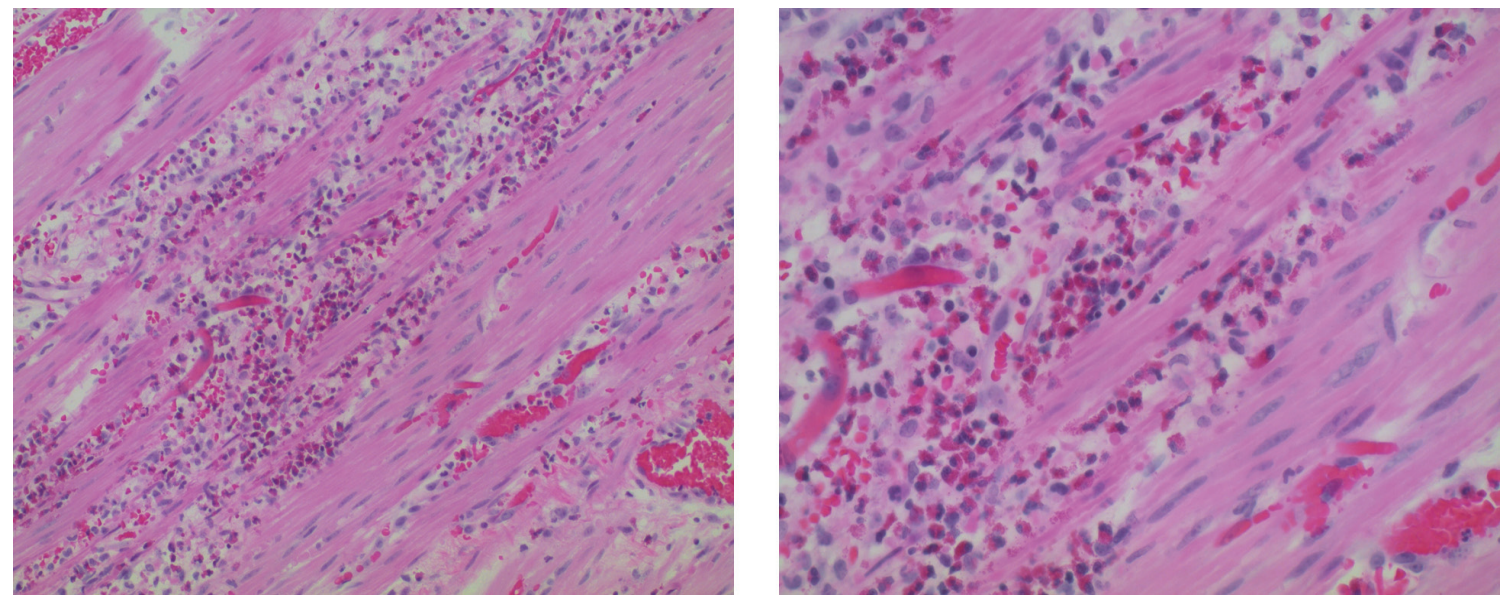

Figure 2. Surgical pathology: dense infiltrate of eosinophils in the muscularis propria suggests EG. 
mately $57.5 \%$ have mucosal, $30 \%$ muscular and $12.5 \%$ (sub) serosal disease, respectively [7]. Although the disease may affect all ages, typical presentations are in the third through fifth decades. EG tends to have an equal gender distribution, $50 \%$ of patients may be atopic and $50 \%$ may have a history of food intolerance of allergy [8]. Mucosa-predominant disease is manifested as non-specific abdominal discomfort, malabsorption and protein-losing enteropathy [9], whereas muscularis-predominant disease leads to bowel wall thickening and intestinal obstruction. Cramping and abdominal pain associated with nausea and vomiting occur frequently. It can also present as an obstructing cecal mass or intussusception $[1,9]$. In contrast, subserosa-predominant disease more commonly results in eosinophilic ascites and markedly elevated counts of eosinophils in peripheral blood [9]. Peripheral eosinophilia occurs in a variable number of EG patients (20$90 \%$ ), and is not a reliable prerequisite for diagnosis [10]. The endoscopic appearance in EG is non-specific, including erythematous, friable, nodular and occasional ulcerative changes [6]. In the muscular form, endoscopy may appear normal. Most EG cases have been diagnosed on surgical, full-thickness biopsy or resection performed for obstruction or suspicion of malignancy $[7,11]$. Radiographically, there is no pathognomonic appearance of EG, the appearance being identical to Crohn's enterocolitis, with thickening and flattening of the valvulae conniventes (most common), strictures, filling defects, ulceration, polypoid lesions and rigidity of the ileocecal valve [10]. The mainstay of treatment in non-obstructive disease is a trial of dietary avoidance of food allergens, but if the symptoms persist or are severe, then corticosteroid therapy is recommended [7,9]. Prednisone at a dose of 20 - $40 \mathrm{mg}$ daily for 8 weeks can yield a good response. Budesonide is an alternative steroid with fewer systemic side effects [7]. Surgical treatment is required for patients with intestinal perforation and/or obstruction $[2,10]$.

In our case, the patient had small bowel obstruction due to a stricture and small bowel wall thickening caused by EG requiring surgical intervention. The patient was followed up later on with complete resolution of his symptoms.

In conclusion, eosinophilic enteritis should be considered in the differential diagnosis of ileitis. Patients, with muscular form of EG, usually present with symptoms of intestinal obstruction due to small bowel wall thickening and rarely stricture formation. Surgery is the treatment of choice in the presence of obstruction. To our knowledge, very few cases with this entity have been reported.

\section{Conflict of Interest}

The authors declare no conflict of interest.

\section{References}

1. Ingle SB, Hinge Ingle CR. Eosinophilic gastroenteritis: an unusual type of gastroenteritis. World J Gastroenterol. 2013;19(31):5061-5066.

2. Oh HE, Chetty R. Eosinophilic gastroenteritis: a review. J Gastroenterol. 2008;43(10):741-750.

3. Koutroubakis IE. Eosinophilic gastroenteritis; the Greek experience. Annals of Gastroenterology. 2002;15(2):104-105.

4. Mendez-Sanchez N, Chavez-Tapia NC, Vazquez-Elizondo G, Uribe M. Eosinophilic gastroenteritis: a review. Dig Dis Sci. 2007;52(11):2904-2911.

5. Antunez ID, Costas PJ, Jesus RD and Baez MTF. Ascites de novo: an unusual presentation of eosinophilic gastroenteritis. Am J Gastroenterol. 2003;98:S168.

6. Kshirsagar AY, Jagtap SV, Kanojiya RP, Langade YB, Shinde SL, Shekhar N. Eosinophilic enteritis presenting as a rare cause for ileo-ileal intussusception. World $\mathrm{J}$ Gastroenterol. 2007;13(47):6444-6445.

7. Yan BM, Shaffer EA. Primary eosinophilic disorders of the gastrointestinal tract. Gut. 2009;58(5):721-732.

8. Gonsalves N. Food allergies and eosinophilic gastrointestinal illness. Gastroenterol Clin North Am. 2007;36(1):75-91, vi.

9. Wolpin BM, Weller PF, Katz JT, Levy BD, Loscalzo J. Clinical problem-solving. The writing on the wall. N Engl J Med. 2009;361(14):1387-1392.

10. Shweiki E, West JC, Klena JW, Kelley SE, Colley AT, Bross RJ, Tyler WB. Eosinophilic gastroenteritis presenting as an obstructing cecal mass-a case report and review of the literature. Am J Gastroenterol. 1999;94:3644-3645.

11. Mori A, Enweluzo C, Grier D, Badireddy M. Eosinophilic gastroenteritis: review of a rare and treatable disease of the gastrointestinal tract. Case Rep Gastroenterol. 2013;7(2):293-298. 